\title{
Ein Zellenboden am Kilimanjaro - Beobachtungen mit Hilfe der Nahbereichsfotogrammetrie
}

Um die Jahreswende 1970/71 führte das Geographische Institut der Universität Zürich eine Forschungsexpedition zum Kilimanjaro durch. Wir arbeiteten hauptsächlich in der Sattelregion zwischen Kibo und Mawenzi. Im Rahmen von Untersuchungen zum subnivalen Formenschatz tropischer Hochgebirge (FURRER und FREUND, 1973, S. 180 ff.) bot sich mit die Möglichkeit, eine Reihe fotogrammetrischer Aufnahmen zu machen. Sie sollten mithelfen, nicht nur das Aussehen gemusterter Böden zu erfassen, sondern auch den Wechsel der Oberflächengestalt.

Hauptsächlicher formenbildender Vorgang im Untersuchungsgebiet ist die Solifluktion; ihre Definition übernehme ich von TROLL (1944, S. 565 f): «Solifluktion im weitesten Sinn ist die Erscheinung, daß unter der Wirkung langdauernder, jahreszeitlicher oder kurzdauernder, sich häufig wiederholender bis allnächtlicher Gefrornis des Bodens eine lebhafte Verlagerung der Bodenteilchen stattfindet, die sich auf ebenem Gelände in der Bildung von Bodenstrukturen oder Bodentexturen (Frostgefügeböden), auf geneigtem Gelände auch bei ganz geringem Gefälle in einem beträchtlichen hangabwärts gerichteten Massentransport (Frostbodenversetzung) äußert.»

In der Sattelregion des Kilimanjaro stellten sich Frostwechsel praktisch täglich ein und bewirkten - genügend Wasser im Boden vorausgesetzt - Solifluktion. Die dabei jeweils bewegten Massen sind zwar vergleichsweise recht gering: einerseits, weil die nächtlichen Fröste nur wenige Zentimeter in den Boden eindringen und nur begrenzt Wasser aus tieferen Horizonten emporgesogen wird; anderseits vergehen zwischen Auftauen - und damit überhaupt erst möglicher Bewegung - und dem Austrocknen der Oberfläche meist nur wenige Stunden.

\section{Problemstellung}

Die Untersuchungen richteten sich darauf, festzustellen, ob sich durch Solifluktion bedingte Massenverlagerungen an auftauenden Zellenböden erfassen und zeigen lassen, und wie weit quantitative Ergebnisse eine qualitative Beobachtung sinnvoll erweitern können. Außerdem wollte ich die Oberflächenveränderung graphisch anschaulich und instruktiv darstellen.

\section{Die Nahbereichsfotogrammetrie als Aufnahme- und MeBverfahren}

Zur Illustration von Feldbeobachtungen ist die Fotografie ein wichtiges Hilfsmittel: Sie belegt und dokumentiert das Beobachtete. Der Informationsgehalt der üblichen Einzelaufnahmen mit ihren zweidimensionalen Darstellungen ist jedoch gegenüber der Natur im allgemeinen gering - es fehlt der Raumeindruck. Die Stereofotogrammetrie hingegen macht sich die menschliche Fähigkeit des räumlichen Sehens zu Nutze: Von einem Gegenstand werden von zwei ihrer gegenseitigen Lage nach genau bekannten Punkten fotografische Bilder aufgenommen. Dabei verwendet man Präzisionskameras, deren Abbildungsgeometrie und Aufnahmedispositionen auf Hundertstel Millimeter genau bekannt sind.

Indem man dem rechten und dem linken Auge die entsprechenden Bilder gleichzeitig zeigt, kann man das räumliche Modell des Aufgenommenen wieder erzeugen. Am einfachsten geschieht dies mit einem (Spiegel-) Stereoskop, das sich besonders auch zum ersten Durchmustern der Bilder eignet. Wenn man den dreidimensionalen Aufnahmegegenstand exakt ausmessen und grafisch darstellen will, benötigt man spezielle Auswertegeräte, die sogenannten Stereoautographen (Abb. 1).

In meinen Untersuchungen an Solifluktionsformen mit Hilfe der Nahbereichsfotogrammetrie (KASPER, 1975) habe ich das Aufnahme- und Auswerteverfahren eingehend beschrieben; hier nur das Wichtigste:

- Die Aufnahmedistanzen der Nahbereichsfotogrammetrie reichen von etwa 1 Meter bis zu mehreren Zehnermetern;

- der zum Aufnahmezeitpunkt herrschende Zustand wird in allen Einzelheiten objektiv festgehalten;

- die bei der Auswertung erreichbare Genauigkeit hängt im wesentlichen von der Aufnahmedistanz und der Basislänge $a b$ : im vorliegenden Beispiel beträgt der mittlere Fehler an Lage und Höhe eines Punktes weniger als $\pm 1 \mathrm{~mm}$;

- unabhängig von der strukturellen Beschaffenheit des Objektes nimmt die Meßaufnahme wenig Zeit in Anspruch;

Dr. Gerhard Kasper, Assistent am Geographischen Institut der Universität Zürich, Blümlisalpstraße 10, 8006 Zürich. 
Abb. 1: Stereoautograph WILD A-9 mit Zeichentisch, Koordinatenregistriergerät, elektrischer Schreibmaschine und Streifenlocher.

Abb. 2: Stereokamera C-40 mit Spezialstativ für Senkrechtaufnahmen.

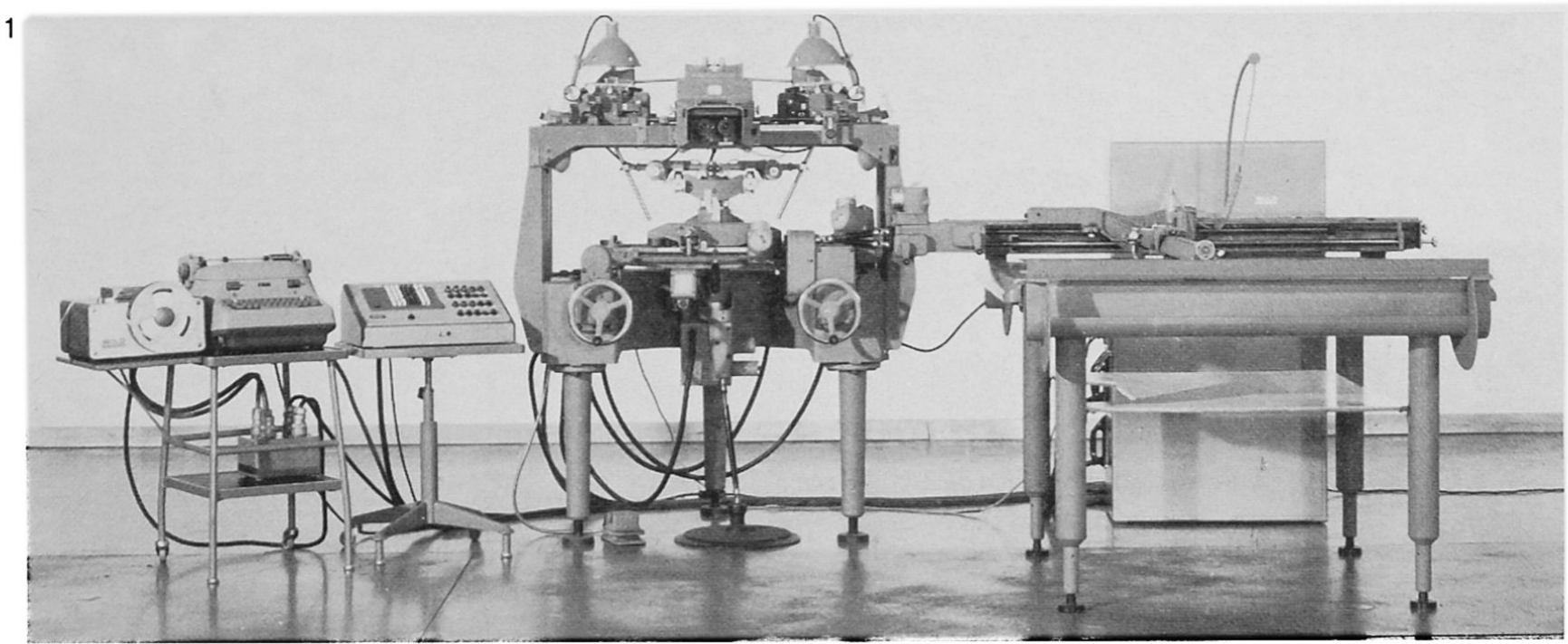

- man muß die Objekte nicht betreten, berühren oder sonstwie verändern, daher kann man insbesonders langsame Zustandsänderungen ungestört erfassen;

- unter Umständen störende äußere Einflüsse (Witterung, Tageszeit, Helligkeit) spielen keine so große Rolle wie bei zeitaufwendigen Feldarbeiten; außerdem kann man ohne Zeitdruck arbeiten;

- gegenüber Einzelbildern sagen Stereobilder wesentlich mehr aus;

- allerdings sind die Aufnahme- und Auswertegeräte verhältnismäßig teuer, so daß sie nicht allgemein verfügbar sind. Meines Erachtens lassen sich jedoch gerade an Hochschulen in Zusammenarbeit mit Instituten für Geodäsie/Fotogrammetrie Lösungen finden, die auch für Geomorphologen die Fotogrammetrie als Meßmethode aufschließen.

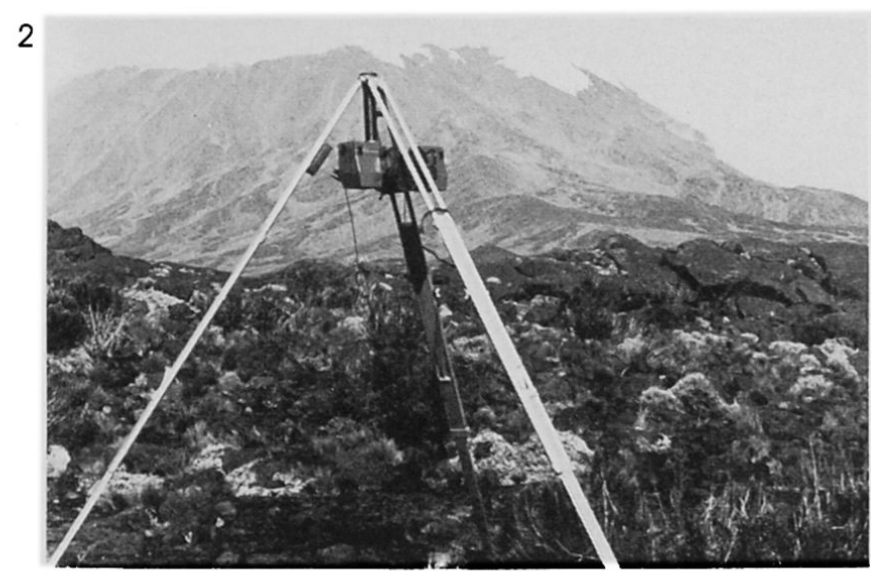

\section{Aufnahme- und Auswerteinstrumente}

Für die Aufnahmen verwendete ich eine Stereomeßkammer Wild C-40 (Basisabstand der beiden Aufnahmeachsen $40 \mathrm{~cm}$ ); sie war an einem Rohr zwischen den Beinen eines Dreibeinstatives aufgehängt; ihre Aufnahmerichtungen zeigten senkrecht nach unten (Abb. 2). Ausgewertet habe ich die Stereomodelle in einem Autographen Wild A-9 mit angekuppeltem Zeichentisch (Abb. 1).*)

\section{Das Untersuchungsfeld}

Der aufgenommene Zellenboden (Abb. 3) ist Teil eines ausgedehnten, locker zusammenhängenden Struktur- und Texturbodenareals in der Sattelregion zwischen Kilimanjaro und Mawenzi; er liegt auf etwa $4300 \mathrm{~m}$ ü. M.; in der nordexponierten Fläche betrug die durchschnittliche Hangneigung $5^{\circ}$.

Die der Bildung der «tropischen Kleinformen» (TROLL, 1944, S. 606, 655) höchst förderlichen, ausgeprägten Tagesschwankungen der Temperatur und Frostwechsel, welche das ganze Jahr über wirksam sind, haben FURRER und FREUND (1973, S. 184f.) (27. 12. 1970 bis 7.1.1971) vom selben Untersuchungsgebiet zusam-

*) Ich danke dem Institut für Geodäsie und Fotogrammetrie der ETHZ für die Kameraausrüstung sowie die zur Verfügung gestellten Auswertegeräte. 
Abb. 3: Zellenboden mit angetauten «Fließziegeln»; Senkrechtaufnahme mit Stereomeßkammer Wild C-40; Gesamtlänge des Maßstabes $2 \mathrm{~m}$; der Pfeil zeigt hangabwärts. Kilimanjaro-Sattel, ca. 4300 m ü. M.

mengestellt. Hier wird ein Auszug aus diesen Werten wiedergegeben - und zwar vom Vortag der Aufnahmen bis zu deren Abschluß am 2.1. Außer den Terminbeobachtungen liegen leider keine Temperaturwerte vor (Tabelle 1).

Tagsüber herrschten auf dem gesamten vegetationsfreien Aufnahmefeld etwa gleiche Besonnungsbedingungen, wenn man vom Schattenwurf vereinzelter aus dem Feinmaterial herausragender Steine absieht.

\section{Die fotogrammetrischen Aufnahmen}

Am 30. 12. 1970 fielen etwa $5 \mathrm{~cm} \mathrm{Naßschnee,} \mathrm{der} \mathrm{auch}$ den Silvester überdauerte und erst am Nachmittag des 1. 1. 1971 langsam abschmolz; darauf begann am 2. Januar die Aufnahmereihe.

Wir verspreizten das Dreibeinstativ schon am Vorabend über dem Zellenboden und hängten die Stereokammer, lotrecht nach unten gerichtet, daran auf. Mit einem Theodolit überprüfte ich die Stabilität der Aufstellung von anstehendem Fels aus. Nur so hätte ein allerdings eher unwahrscheinliches - völlig gleichmäßiges Einsinken aller drei Stativbeine erkannt werden können; jede andere Lageänderung (Drehung, Kippung) der Aufnahmebasis jedoch könnte man an den zwei Röhrenlibellen, mit denen man die Kamera ausrichtet, ablesen und korrigieren. Während des ganzen Versuches mußte das Instrument aber nicht nachjustiert werden; eine Tatsache, die sich einerseits aus der festen Verankerung der Stativspitzen erklärt, anderseits auch für die Stabilität des Instrumentariums spricht.

Als Einpaß- und Bezugspunkte für die spätere Auswertung dienten Marken auf Steinen, deren Lage sich - wie die geodätische Prüfung zeigte, während der gesamten Meßdauer nicht änderte.

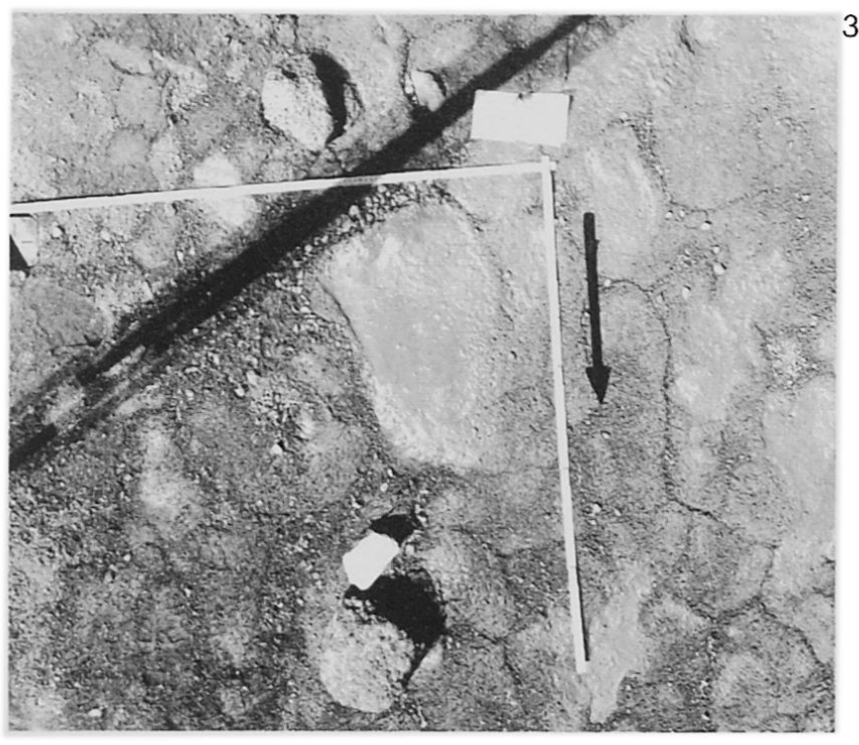

\section{Die zeichnerische Auswertung}

Die fotogrammetrische Auswertung mit Höhenkurven und Umrißlinien erfolgte mit einem Stereoautographen Wild A-9 im Zeichenmaßstab 1:5; als Äquidistanz der Höhenkurven wählte ich $1 \mathrm{~cm}$. Im unmittelbaren Bereich des «Fließziegels» allerdings erwiesen sich Zwischenkurven $(1 / 3 \mathrm{~cm})$ als nötig, erst sie veranschaulichten die Feinheiten der Form und deren Veränderungen.

Neben den vier Zeichnungen im Grundriß (Figuren 1-4) ziehen über alle Formen an den gleichen Stellen Längsprofile: Die Kurven der ersten und letzten Aufnahme sind in Figur 5 wiedergegeben. Die Umrisse des Fließziegels sind kräftig ausgezogen, desgleichen die mit Raster belegten Steine am oberen und unteren Bildrand.

Tabelle 1 Temperaturen und Luftfeuchtigkeit beim Untersuchungsfeld, 1. 1.-2. 1. 1971

\begin{tabular}{|c|c|c|c|c|c|}
\hline Zeit & $\begin{array}{l}150 \mathrm{~cm} \\
\text { über Boden }\end{array}$ & $\begin{array}{l}10 \mathrm{~cm} \\
\text { über Boden }\end{array}$ & $\begin{array}{l}5 \mathrm{~cm} \text { unter } \\
\text { Bodenoberfläche }\end{array}$ & $\begin{array}{l}10 \mathrm{~cm} \text { unter } \\
\text { Bodenoberfläche }\end{array}$ & $\begin{array}{l}\text { Luftfeuchtig- } \\
\text { keit in } \%\end{array}$ \\
\hline 1.1. $19.00 \mathrm{Uhr}$ & $+1.5^{\circ}$ & $+3.0^{\circ}$ & $+7.5^{\circ}$ & $+7.0^{\circ}$ & 25 \\
\hline 2. 1. $07.00 \mathrm{Uhr}$ & $-2.0^{\circ}$ & $-2.0^{\circ}$ & $+2.0^{\circ}$ & $+3.0^{\circ}$ & 67 \\
\hline 13.00 Uhr & $+9.0^{\circ}$ & $+11.5^{\circ}$ & $+10.5^{\circ}$ & $+8.0^{\circ}$ & 29 \\
\hline 19.00 Uhr & $0.0^{\circ}$ & $+1.5^{\circ}$ & $+7.0^{\circ}$ & $+7.5^{\circ}$ & 16 \\
\hline
\end{tabular}


Abb. 4-7: Fotogrammetrisch untersuchter Fließziegel; Aus-

schnitt aus den Meßaufnahmen mit der Stereomeßkammer

Wild C-40; Aufnahmedatum und -zeiten: 2. Januar 1971

07.15, 08.45, 09.20, 11.00. Kilimanjaro Sattel, ca. 4300 m ü. M.
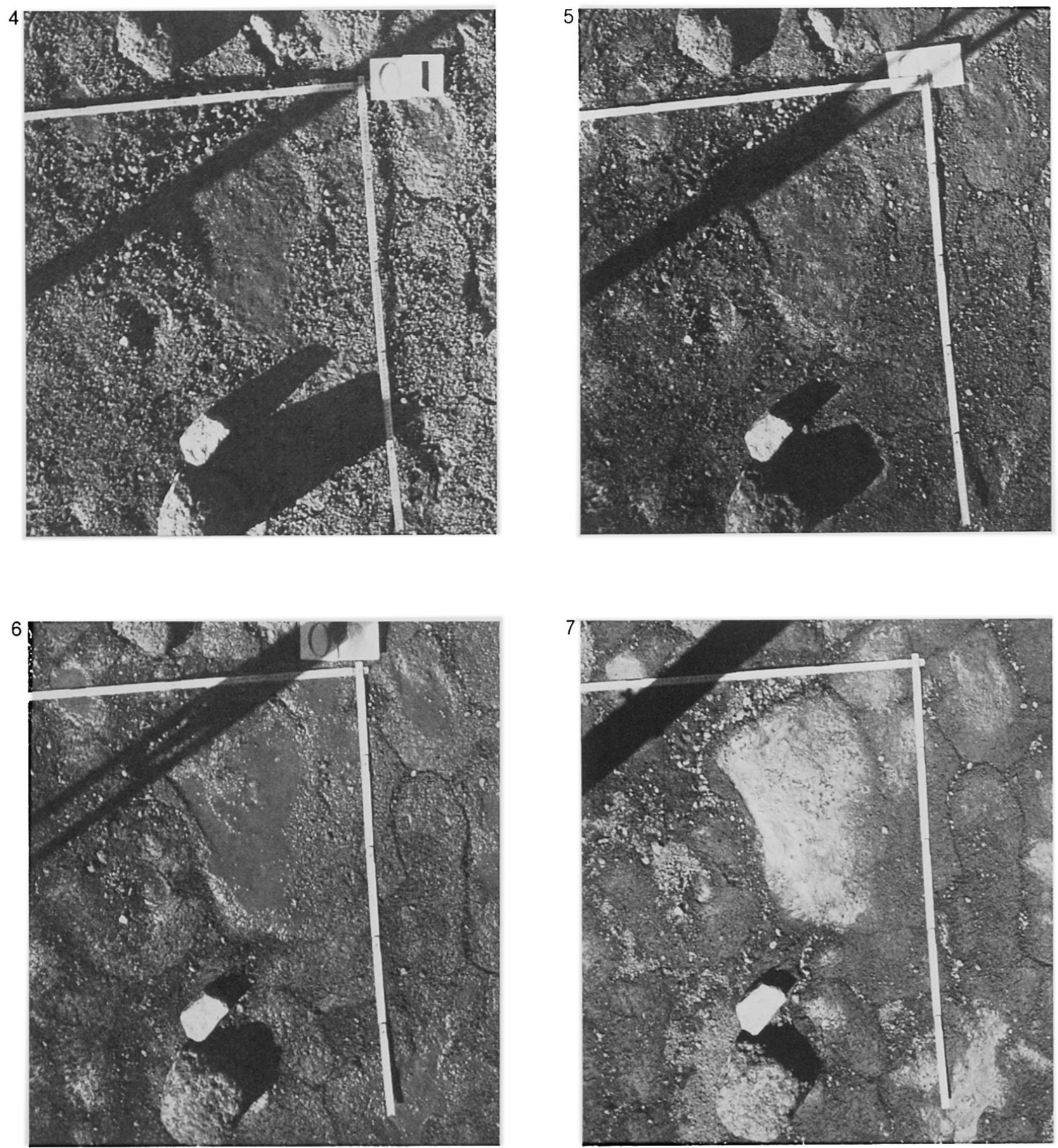


\section{Vergleich zwischen den Fotografien und den fotogrammetrischen Auswertungen, ergänzt durch Feldbeobachtung}

\section{Aufnahme Nr. 1 (Abb. 4)}

Die Oberfläche der Zunge ist zusammenhängend gefroren, die "Eisschicht» netzartig strukturiert; Risse und Spalten der Umgebung, die den Texturboden in Zellen gliedern, sind durch Kammeis teilweise verwischt; die Temperaturen an der Bodenoberfläche und $10 \mathrm{~cm}$ über Grund liegen bei $-2.0^{\circ} \mathrm{C}$.

Neben dem beobachteten Erdziegel finden sich noch weitere, nicht mit Kammeis bestandene Flecken, auf denen sich - wie die späteren Aufnahmen zeigen werden - ebenfalls Durchtränkungsfließen abspielen wird. $\mathrm{Ob}$ zwischen der Oberflächenbeschaffenheit in gefrorenem Zustand (zusammenhängende «Eishaut» oder Kammeis) und dem Durchtränkungsfließen ein direkter Zusammenhang besteht, kann allein aufgrund der Feldbeobachtungen nicht mit Sicherheit gesagt werden. Möglicherweise handelt es sich um eine Erscheinung, die auch beim künstlich erzeugten Kammeis im Labor auftritt: Wird nämlich eine Bodenprobe so mit Wasser getränkt, daß schließlich gerade nichts mehr versickern kann, so entsteht auf skelettarmer Feinerde eine mehr oder minder glatte Oberfläche. Temperaturbedingungen, unter welchen bei einer in Vorversuchen gefundenen idealen Feuchtigkeitskonzentration im Bodenmaterial jedesmal Kammeis wächst, bewirken auf völlig durchnäßter (nicht aber unter Wasser stehender) Probe eine lückenlose «Eisschicht», die gitterartig von dickeren Eisrippen durchzogen ist. Selbst wenn nach mehrmaligen Frostwechseln auch Wasser verdunstet ist, bleibt die Tendenz zur Kammeisbildung viel geringer als bei einer schon a priori gekrümelten Deckschicht. Setzt man nach dem ersten Auftreten von Nadeleis-«nestern» den Versuch unter gleichbleibenden Feuchteverhältnissen fort, so weitet sich bei jedem Frieren die Kammeisfläche aus - nach dem Abtauen nimmt die gekrümelte Fläche entsprechend zu. Platzregenartiges Begießen der Probe führt die lockere Oberfläche in einen breiigen, glatten Zustand zurück, wo Kammeis nur wieder sehr schwer angreifen kann: Es können sich kaum Eiskeime bilden, ohne daß sie sogleich mit ihren Nachbarn zusammenwachsen und so eine Art Eispanzer formen. Wo die Grenzwerte der Kammeisbildung liegen, muß noch abgeklärt werden.
Bisher darf jedenfalls folgendes ausgesagt werden: Feinmaterial mit glatter Bodenoberfläche hindert das Aufkommen von Kammeis; krümelige Flächen neigen unter vergleichbaren Feuchtigkeitsverhältnissen eher zur Nadeleisbildung.

Wenn die «Eisschicht» auftaut, verschwinden auch die eingeschlossenen Blasen, welche auf Abbildung 6 als weiße Kreise erkennbar sind. Sie scheinen in erster Linie durch Frosteinflüsse entstanden zu sein, wie die von FURRER (1954) und FREUND (1972) untersuchten Frostblasen in Erdziegeln; bei letzteren handelt es sich jeweils um ausgesprochen skelettarme Feinerde mit wenigen Zentimetern Mächtigkeit. FREUND fand frühmorgens in gefrorenen Erdziegeln eines Polygonbodens am Kilimanjaro in einigen Blasen auch kugelförmige Eiskörnchen, welche er als Ursache für die Genese der Blasen betrachtet. Im vorliegenden Fall hingegen ist die ins Fließen geratene Schicht nur wenige Millimeter mächtig; das Material ist derart stark durchnäßt, daß die Bildung reiner, isoliert auftretender Eiskeime und Eiskörner - wie oben beschrieben kaum vorkommen kann. Es handelt sich wahrscheinlich um Bodenluft, welche infolge der Einstrahlung erwärmt wird, aufsteigt und in der breiigen Schlammschicht Blasen wirft (siehe dazu auch Ausführung bei Aufnahme Nr. 4, Abbildung 7).

Auf der fotografischen Aufnahme erscheint zwischen der Kammeisfläche und dem Erdziegel mit seiner «Eishaut» ein überaus deutlicher struktureller Unterschied der Oberflächen. Das Streiflicht der Morgensonne verstärkt noch den plastischen Eindruck. Wenn nun in der fotogrammetrischen Auswertung der Aufnahme Nr. 1 (Abb.4, Fig. 1) die Höhenlinien trotzdem in gleicher Charakteristik verlaufen, so hat das zwei Gründe: 1. erst die stereoskopische Betrachtung zeigt, $\mathrm{da} ß$ die vertikale Zergliederung selbst der Kammeisfläche viel geringer ist, als man auf der Einzelaufnahme vermuten möchte (Schräglicht!); 2. das «Auflösungsvermögen» der linienweisen Kartierung reicht in diesem Maßstab nicht aus, jede kleinste Vertiefung wiederzugeben: Die grafische Auswertung generalisiert also. (Durch punktweises, digitales Kotieren lieBen sich auch solche minimale Unregelmäßigkeiten erfassen, aber kaum in allen Einzelheiten anschaulich darstellen. [KASPER, 1975]). 

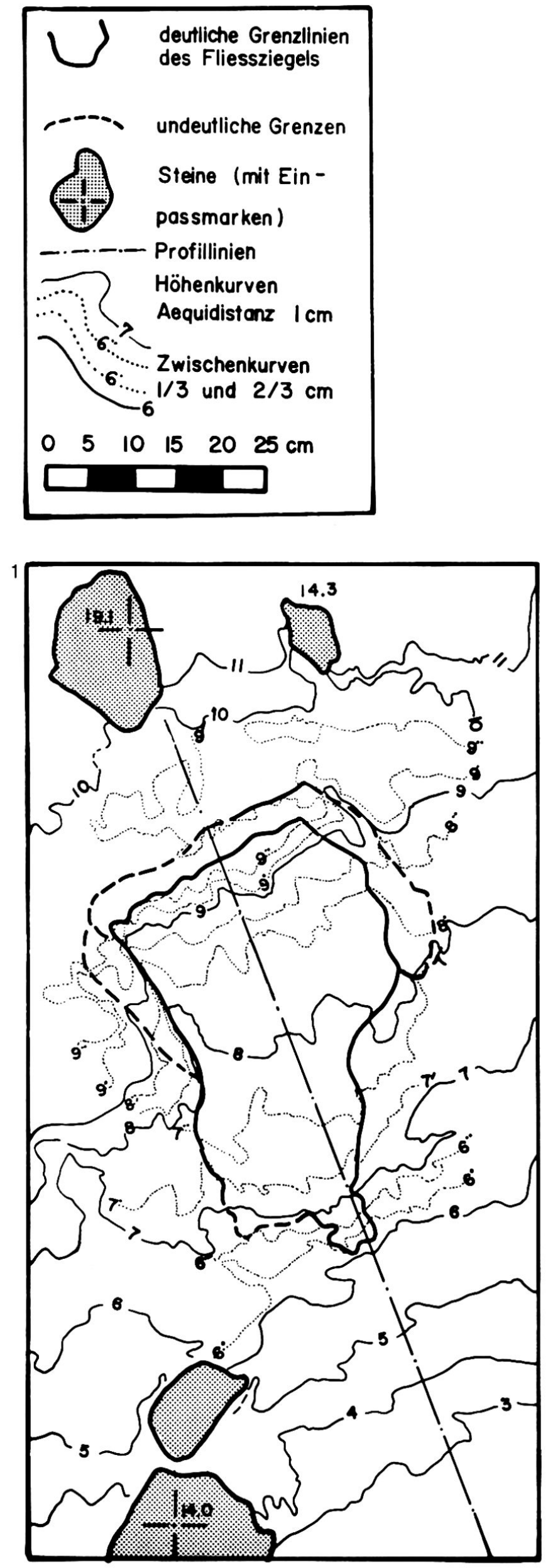

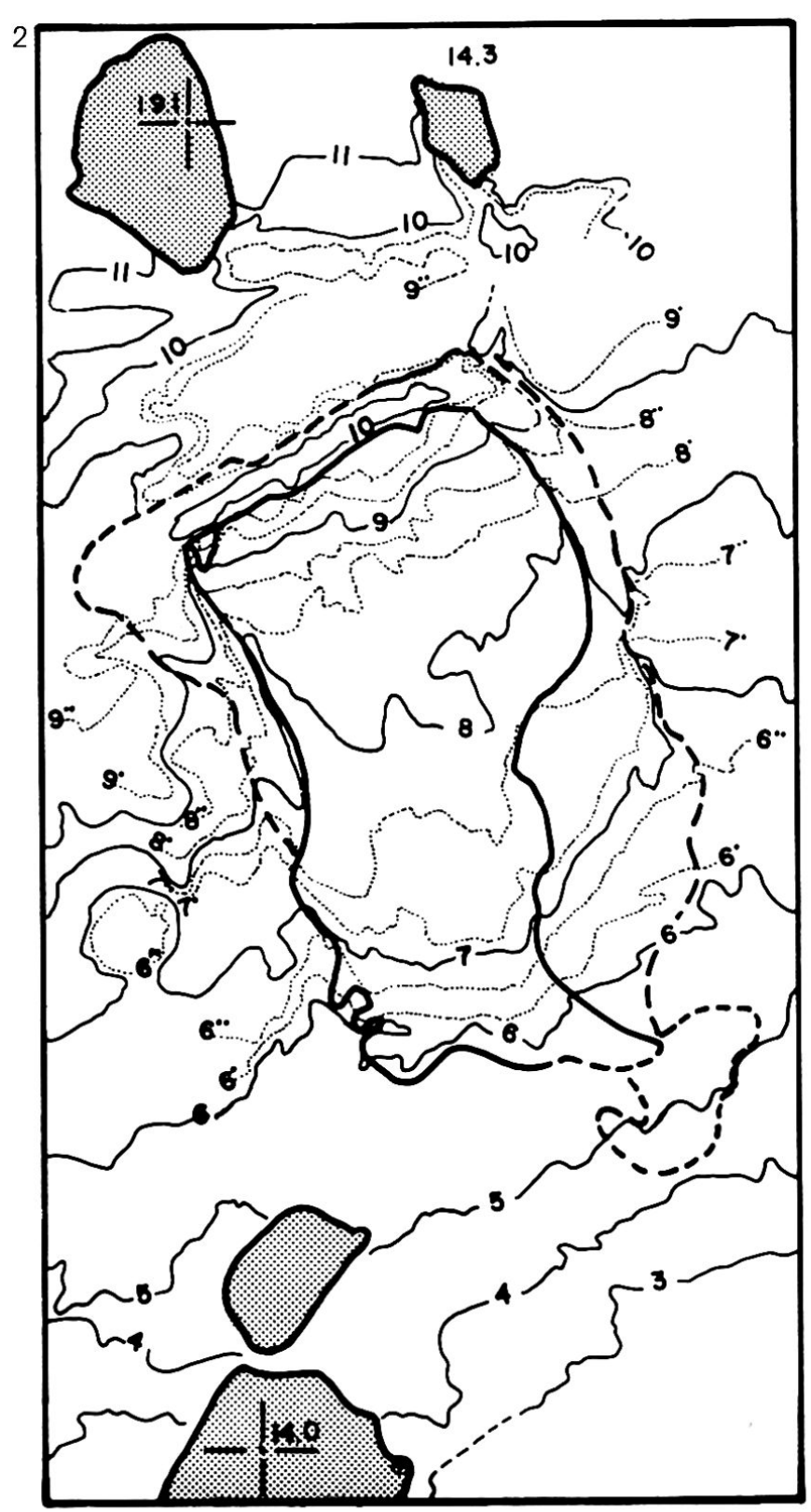
sich langsam auf; Bodentemperatur $+1.5^{\circ} \mathrm{C}$. Am längsten bleiben die dickeren Rippen des Netzwerkes erhalten, während die Eisplättchen dazwischen abschmelzen, das unter ihnen liegende Material immer stärker durchfeuchten und schließlich verflüssigen.
Die Netzstruktur der oberflächlichen «Eishaut» löst 
Fig. 1-4: Fotogrammetrische Auswertung der Fließziegel aus den Abbildungen 4-7 mit Höhenkurven und Umrißlinien.

Fig. 5: Längsprofile über den Fließziegel aus den fotogrammetrischen Auswertungen der Abbildungen 4 und 7. Die Lagen der Profile sind in Fig. 1 und 4 eingetragen. Maschenweite des Rasters $5 \mathrm{~cm}$.

Am untersten Ende der Zelle fließt bereits Tauwasser, durch Schwebepartikel nur wenig getrübt, ab. Es unterspült die schwach hangabwärts gerichteten Kammeisnadeln. Dadurch tauen diese an der Basis, brechen ab und stürzen überwiegend in der Richtung des Gefälles. Die letzten Aussagen fußen auf Feldbeobachtungen: diese werden auch beim angewendeten fotogrammetrischen Aufnahme- und Meßverfahren weiterhin

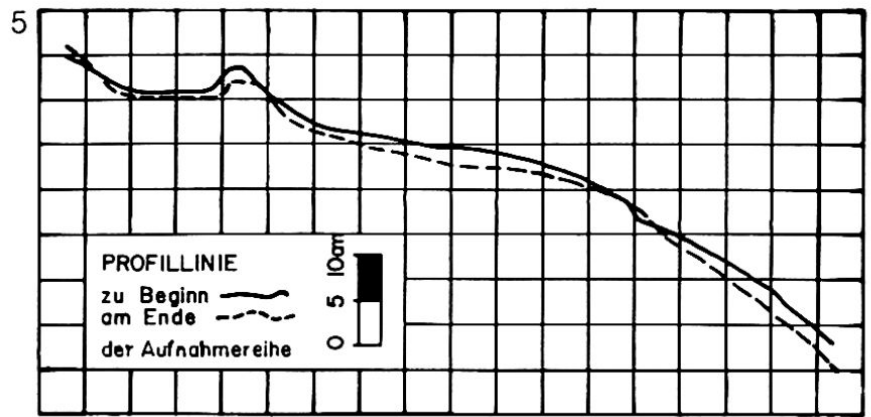

wesentlicher Bestandteil geomorphologischer Untersuchungen bleiben.

In der fotogrammetrischen Auswertung (Fig. 2) deutet sich bereits an, wie sich die angetaute Oberfläche auf der - hangabwärts betrachtet - linken Seite ausweitet. Vergleicht man die Höhenkurven mit Darstellung 1, so sind noch keine durch Massenverlagerung hervorgerufenen Oberflächenveränderungen erkennbar.
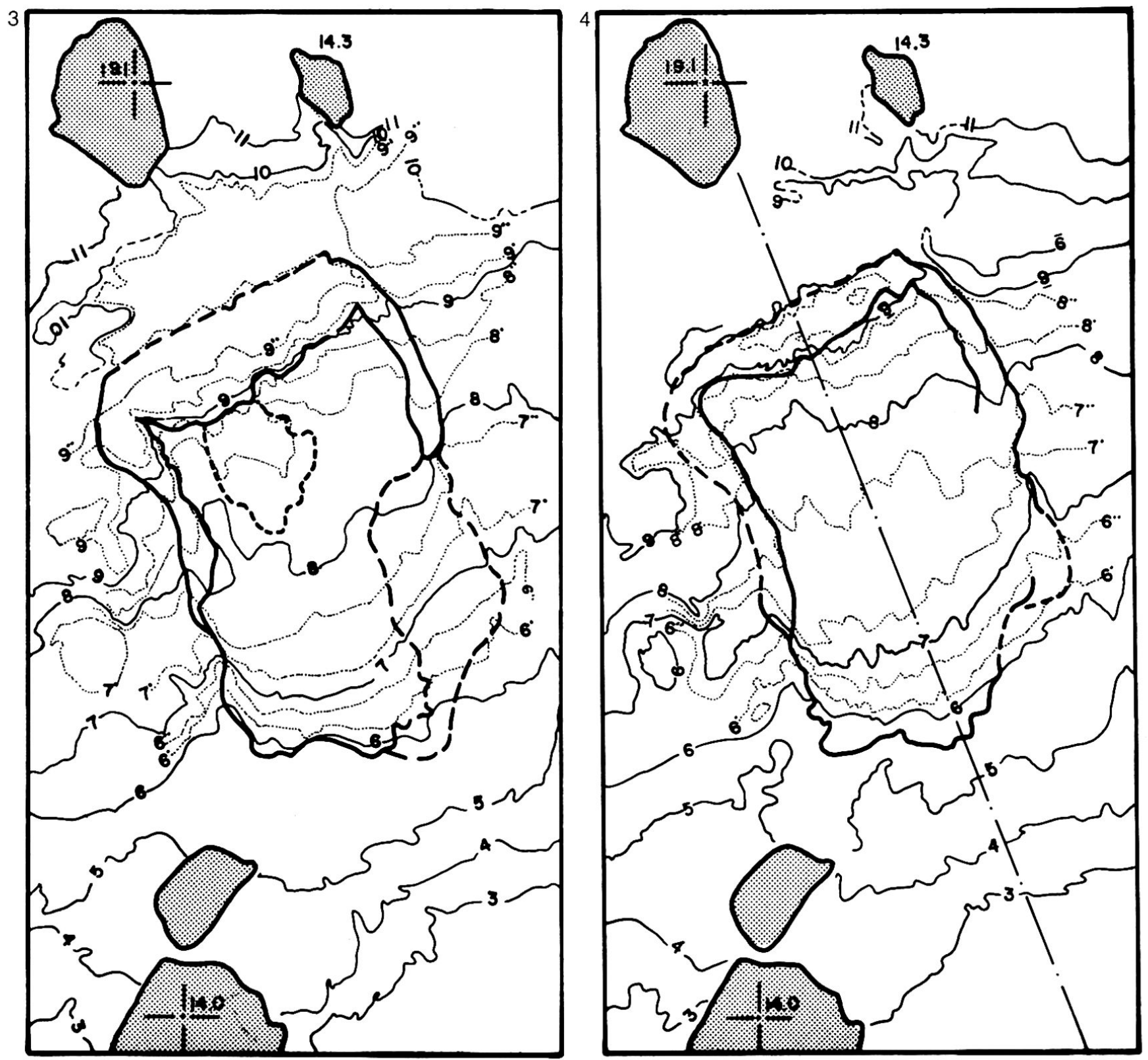


\section{Aufnahme Nr. 3 (Abb. 6)}

Zumindest an der Oberfläche ist der Erdziegel vollständig aufgetaut. Nur im oberen Teil ist vorläufig noch ein kleiner gefrorener Sockel stehengeblieben, dessen Oberfläche mit aufsteigenden und zerplatzenden Bläschen dicht bedeckt ist. An den Rändern - besonders in Gefällsrichtung gesehen links - gewinnt die Durchfeuchtungszone an Raum und ebnet dabei die anfänglich gekrümelte, ehemals mit Kammeis bestandene Fläche ein.

Von einer Materialverlagerung hangabwärts zeugen die Höhenkurven des mittleren und unteren Zungenteils, welche alle in Gefällsrichtung abwärts gerückt sind. Dort unten wird auch deutlich, daß die aufgetaute Oberfläche der Fließzunge viel glatter ist als die Umgebung. Im jetzigen, fließaktiven Zustand dehnt sich die vom praktisch unbewegten Umgebungsmaterial abgrenzbare Fläche am weitesten aus.*)

\section{Aufnahme Nr. 4 (Abb. 7)}

Zur Zeit der letzten Aufnahme war die ganze Oberfläche trocken. $10 \mathrm{~cm}$ über dem Boden betrug die Temperatur knapp $10^{\circ}$, am Boden $6^{\circ}$. Unter den Ausführungen zur Aufnahme 1 (Abb.4) ist eine Möglichkeit der Blasenbildung bei schlammiger Fließerde bereits angedeutet (aufsteigende Bodenluft wirft Blasen). In der allmählich austrocknenden und immer zäherflüssigen bis festen Masse bildet die aufsteigende Luft schließlich keine Blasen mehr: die letzten, gerade noch an die Oberfläche gelangten, zerplatzen; zurück bleiben runde, kraterartige Eintiefungen.

Durch Austrocknung schrumpft der Erdziegel, seine Oberfläche strukturiert sich wieder stärker - das zeigt ein Vergleich zwischen den Höhenkurvenbildern der Figuren 3 und 4.

Betrachtet man die Isohypsen im Bereich der 7-cm und 8-cm-Kurven, so kann man aus deren Verschiebung annehmen, daß die hangaufwärts gerückten Kurven $72 / 3$ und $8.0 \mathrm{~cm}$ auf einen Materialschwund im zentralen Teil des Fließziegels deuten. Dieser rührt aber nicht nur aus der Verdunstung von Bodenwasser her, das zeigen die Linien 6.0 und $7.0 \mathrm{~cm}$ : Zur Zeit der Aufnahme war auch das untere Zungenende staubtrocken, aber das Wasser hatte offenbar während der

*) Für die Flächenmessungen diente ein Präzisionsplanimeter Coradi SRP.
Fließphase (zwischen den Aufnahmen 1 und 4) viel Festmaterial vom Zungenursprung ans untere Ende gespült - das belegen die Profile (Fig. 5) und die Höhenlinien.

Die Fläche, die sich als potentiell fließaktiv von der Umgebung abgrenzen läßt, hat sich gegenüber Aufnahme Nr. 1 um mehr als 1 Quadratdezimeter ausgedehnt (Nr. 1: $5.85 \mathrm{dm}^{2}$, Nr. 4: $7.13 \mathrm{dm}^{2}$ ).

\section{Zusammenfassung}

An einem schwach geneigten Zellenboden vom Kilimanjaro habe ich versucht, Bewegungen und Veränderungen der Oberflächengestalt zu erfassen und darzustellen.

Als wichtigstes Hilfsmittel diente die Nahbereichsfotogrammetrie mit stereoskopischen Aufnahmen: Sie ermöglichte es, verschiedene Zustände des Fließbodens festzuhalten und später zu Hause in Details auszumessen und grafisch darzustellen. Stereobilder können zwar die Natureindrücke nicht ersetzen; mit ihrer Hilfe kann man aber Untersuchungen und Messungen anstellen, die am Objekt selbst unmöglich wären.

Höhenkurven, Umrißlinien und Profile zeigen, wie sich ein auftauender und allmählich austrocknender Fließziegel bewegt und gibt Anhaltswerte für Materialverlagerungen hangabwärts.

\section{Literaturverzeichnis}

FREUND, R.: Vergleichende Betrachtungen von Kleinformen der Solifluktion im Raume Mittelbünden (Schweiz), auf Westspitzbergen und am Kilimanjaro. 1972, Diss., Univ. Zürich.

FURRER, G.: Solifluktionsformen im Schweizerischen Nationalpark. 1954, Diss. Univ. Zürich.

FURRER, G. und FREUND, R.: Beobachtungen zum subnivalen Formenschatz am Kilimanjaro. 1973, Z. Geomorph. N.F. Suppl. Bd. 16.

KASPER, G.: Untersuchungen an Solifluktionsformen mit Hilfe der Nahbereichsfotogrammetrie. 1975, Diss. Univ. Zürich.

TROLL, C.: Strukturböden, Solifluktion und Frostklimate der Erde. 1944, Geol. Rdsch. Bd. 34, Heft 7/8. 